\title{
Response to the Commentaries on Pseudohallucinations in an Adolescent: Considerations for Diagnosis and Treatment in the Case of "Kate"
}

\section{A Reconceptualization of Pseudohallucinations in the Case of "Kate"}

\author{
MICHAEL A. SHAPIRO, ${ }^{\mathrm{a}, \mathrm{b}}$ REGINA BUSSING, ${ }^{\mathrm{a}}$ \& MATHEW L. NGUYEN ${ }^{\mathrm{a}}$ \\ ${ }^{\text {a }}$ University of Florida Department of Psychiatry, Division of Child and Adolescent Psychiatry \\ ${ }^{\mathrm{b}}$ Correspondence regarding this article should be addressed to Michael A. Shapiro, University of Florida \\ Department of Psychiatry, Division of Child and Adolescent Psychiatry, 8491 NW 39th Ave, Gainesville, FL 32606 \\ Email: mshapiro@ufl.edu
}

\begin{abstract}
In the case study of "Kate" (Shapiro, Bussing, \& Nguyen, 2014), we discussed a case of a 16year-old adolescent who initially presented with auditory hallucinations and secondary delusional thinking. A more thorough examination of the underlying dynamics of the patient and family pointed away from a diagnosis of a psychotic disorder, and the consideration of "pseudohallucinations" - conversion disorder symptoms in the form of psychiatric symptomswas felt key by the treating child psychiatrist in tailoring treatment for Kate and her family. We discussed potential definitions of "pseudohallucinations" and used a psychodynamic formulation as a conceptual framework to provide treatment in the form of cognitive-behavioral therapy, psychodynamic therapy, and family therapy. Kate improved during the course of treatment, and thankfully avoided long-term treatment with antipsychotic medications. In response to three thoughtful and stimulating commentaries on the original case study by Pienkos (2014), Westerman (2014), and Mertin (2014), we discuss other ways in which Kate's case can be conceptualized, and we further explore the phenomenon of non-psychotic hallucinations. We focus on the main thematic elements in these commentaries, namely, Kate's struggle for autonomy and independence as a main driver in symptom formation; the theoretical approach to the case; and the continued focus on the hallucinatory experiences.
\end{abstract}

Key words: adolescent; auditory hallucinations; autonomy; pseudohallucinations; case study; clinical case study

\section{INTRODUCTION}

In our original case study of "Kate," (Shapiro, Bussing, \& Nguyen, 2014), we present a case study of an adolescent female who experiences auditory hallucinations and secondary delusional thinking. After determining the symptoms were not representative of a psychotic disorder, Kate was successfully treated with individual cognitive-behavioral therapy, psychodynamic therapy, and family therapy. The original case study focuses on Kate's hallucinatory experiences as unconscious manifestations of anger and aggression, feelings that 
Kate felt unacceptable and that burdened her with guilt. Therapeutic interventions with both Kate and her family were geared toward preventing the reinforcement of the hallucinations, supporting the healthy and age-appropriate expression of Kate's feelings of anger and aggression, and helping Kate develop a more mature identity within her family system. This led to a more appropriate conceptualization of the diagnosis and treatment, ultimately avoiding longterm treatment with antipsychotic medications and the associated potential side effects.

The phenomenon of hallucinations in children and adolescents has been increasingly described but not fully understood, particularly with the delineation of psychotic and nonpsychotic hallucinations. We received commentaries by three authors-Pienkos (2014), Westerman (2014), and Mertin (2014) — who each provided their own insight from different vantage points, including alternate views of important aspects of the case, different therapeutic modalities from which Kate's situation could be understood and treatment could be tailored, and the significance of such hallucinations in general. Mertin, who has previously written on hallucinations in the child and adolescent population, provides additional thoughts and comments regarding the nature and meaning of hallucinations in children and adolescents, and possible mechanisms by which they are produced. Pienkos and Westerman add to this discussion, and also view Kate's case through the eyes of different therapeutic perspectives, namely philosophical phenomenology and interpersonal defense theory, respectively.

In this reply to the commentaries, we retrospectively look to them for helpful analysis and new insight, trying to highlight both similarities and differences of the various theoretical underpinnings from which an understanding of this case can be sought. Overall, it appears that we and the commentators agree that the individual and unique details of Kate's experience within her family system are necessary to provide the context within which a full and proper formulation of Kate's case can be developed. Specifically, we aim to tie together the common themes of this case, namely the significance of Kate's search for autonomy and independence, as seen through differing but potentially overlapping theoretical perspectives; the significance of the hallucinations themselves; and whether or not the hallucinations should be the focus of diagnosis and treatment.

\section{AUTONOMY AND INDEPENDENCE}

A common theme in the commentaries was Kate's struggle to become more autonomous and independent, and how this struggle was integral to understanding the dynamics of Kate and her family. We certainly agree that encouraging Kate to become more autonomous and independent from her parents was a main goal of treatment, and that more discussion in the original case study regarding this topic would have been beneficial. Mertin agrees that the family history, specifically the parents' infantilizing of Kate, and Kate's struggle for greater autonomy, placed Kate's presentation in a more understandable context. Moreover, in the context of our discussion in the original article of Kate's expression of anger and overcoming her guilt when doing so, Westerman makes an important distinction. He points out that expressing anger and acting autonomously are not one and the same, and greater focus on the attainment of autonomy and independence would be an important consideration. Although these two are related, not all children and adolescents explicitly seek autonomy or independence, and not all are angry if they 
feel these opportunities are limited. As Westerman notes, Kate struggled with both wanting to be autonomous and also wanting to be taken care of by another authority figure.

Westerman's view is that the main conflict and driving force of symptoms were interpersonal issues specifically related to her parents. Westerman posits that Kate was "afraid that if she behaved in ways to ... act in autonomous, independent ways, her parents would neglect/ignore her" (2014, p. 276-277). Interestingly, this theory was tested in reality not with Kate's parents, but with her peer group. The hallucinations were most present around her parents, but initially Kate endorsed hearing them at school as well, and she even talked to her friends about the hallucinations.

The reactions of her friends, however, were much different than that of her parents. Whereas her parents took great interest and concern with the hallucinatory experiences, Kate's friends told her she was "weird" and withdrew from her. Kate then stopped sharing her hallucinatory experiences with her friends, but continued to do so with her parents. Thus, it seems that Kate's friends ignoring her served to work against the purpose of Kate's hallucinations to gain social support, or, in behavioral terms, served to punish Kate's discussing her hallucinations with her friends. In terms of her parents, Kate explicitly said her fear was of upsetting them, which may have brought forth a situation in which Kate would have been neglected. It is questionable whether Kate's main fear was of being neglected, or rather feeling guilty for allowing (or causing, in her mind) harm to befall her parents. This last point is more analogous to the content of her hallucinatory and delusional experiences, believing there was "a conspiracy she had to stop” and that there was a force trying to kill her parents.

However, one important distinction to make is whether Kate's parents reacted to her acting independently, or rather reacted to the results of her acting independently. Kate seemed to feel she was ignored not if she acted independently, but if she was "doing what she was supposed to.” In other words, if homework and chores were completed, and if her grades were where her parents expected, Kate then felt ignored, even if she acted independently to produce these results. If there were different results, such as a slip in academic grades or a failure to complete household chores in a manner deemed sufficient, Kate's parents, particularly her mother, acted critically.

Just as Westerman notes that expressing anger and acting autonomously are not equivalent, neither are acting autonomously and producing desired results. The parents' focus on desired results is in part what drove them to infantilize Kate, as Kate was not trusted to produce these results herself. Westerman notes that the hallucinations were "a defensive way to be independent" (2014, p. 277), but as he also points out, Kate's desire to be independent was wrought with internal conflict and ambivalence. Therefore, the hallucinations could also be viewed as a way to assume the sick role, which is contrary to the concepts of independence and autonomy. However, Kate's fear may have been that after enough frustration and/or criticism from not producing sufficient results, Kate's parents would then turn to neglecting or ignoring her. Therefore, both points of view may be valid. 


\section{THEORETICAL APPROACH TO THE CASE}

We made an explicit choice to conceptualize Kate's case within a psychodynamic framework, as reflected in part by an earlier version of her case study that won the AACAP Rieger Psychodynamic Psychotherapy Award. Therefore, while several other viewpoints and theoretical frameworks are certainly valid and could provide additional insight, there was a concrete reason for focusing our case conceptualizion within a psychodynamic approach. Moreover, psychodynamic and cognitive-behavioral orientations were an important part of the Child and Adolescent Psychiatry Fellowship within which the first author (M.S.) conducted therapy with Kate, and this also influenced the selection of theoretical approach.

In this context, at light, we can address Westerman's concern of focusing too much on Kate's “inner experience” as a result of choosing a psychodynamic viewpoint. Certainly Kate’s relationship with her parents and others are critical to formulating Kate's circumstance. Otherwise, her "outward” experience was quite appropriate, in terms of school performance, socializing with friends, and extracurricular activities. In fact, her intact social functioning was often used not only to quell the family's fears of a psychotic disorder, but also to validate Kate's autonomy that she could take care of herself, and as an observation to her parents that when left to make her own decisions, Kate's choices would result in "good enough" results. However, Kate's inner experience is where Kate indicated she suffered, so it made sense to us that treatment was focused in that arena, in addition to the reasons previously stated.

Westerman points out that we referenced the Lukianowicz (1969) article, which identifies possible "aims and functions" of non-psychotic hallucinations as the sole source of developing a psychodynamic formulation. Along those lines is Westerman's critique of focusing excessively on the hallucinations in regards to a dynamic understanding of Kate and her family. We do agree that Lukianowicz does focus solely on hallucinations, and perhaps a more experienced clinician may have seen that the hallucinations were merely one of many types of symptoms that may have resulted from Kate's complex but not too uncommon family dynamics. With that, we also see many similarities between Lukianowicz's theory and Westerman's interpersonal defense theory: both involve wish-fulfilment, a feared response, a method of avoiding/escaping a feared outcome, and re-living or re-experiencing an event or process: following:

Lukianowicz's (aims and functions of non-psychotic hallucinations include the

1) as an escape mechanism to elude fear/anxiety or from intolerable reality;

2) as a wish-fulfilling mechanism to achieve satisfaction denied in real life;

3) as externalized fear/anxiety, leading to relief;

4) as an expression of intolerable hostile feelings/wishes;

5) as an expression of feelings of guilt or fear of punishment; and

6) as a neurotic reliving of a traumatic event. 
Westerman's interpersonal defense theory includes the following:

1. "the individual's central wished-for interpersonal response;"

2. "a central feared response" that might occur if the wish is pursued;

3. "a specific pattern of defensive behavior that attempts to do two things that are ultimately incompatible - pursue the wish, but also work to avoid the feared outcome;"

4. maintenance of the defensive pattern because even though the person's wish is not realized, he or she does avoid the fear and also realize some positive outcomes; and

5. "negative outcomes distinct from the central fear that the defensive pattern actually promotes" (2014, p. 275-276).

Particularly, "a specific pattern of defensive behavior" that results from the conflict of two seemingly incompatible goals is part of the key for an extrapolation of a dynamic interpretation. This serves as a model for most dynamic conflicts, that is, between the id versus the superego. The recurring discourse breaches are conceptually the more likely outcome if the ego is unable to modulate between the two entities. This is true in Kate's case. The expression of her anger served as an external projection of her internal conflict, which Westerman identifies as autonomy. Westerman's “recurring coordination failures” (2014, p. 276) refers essentially to the repetitive parallel process. Thus Lukianowicz's and Westerman's theories can both be seen to have roots in psychodynamic psychotherapy.

We found Pienkos' viewpoint of philosophical phenomenology insightful, and agree that the main goal of conceptualization should be to "contribute to an understanding of the overall picture of a person's experience" (2014, p. 263). Through Pienkos' lens of phenomenology, the major themes of Kate's internal conflicts are easily seen in the themes of her hallucinations, which could refer to her perception of the hallucinations and her perception of her relationship with her parents:

1) Kate must choose to obey or disobey a seemingly powerful authority;

2) Significant responsibility was placed on her to protect others;

3) Kate felt a sense of intrusion to her private space;

4) Kate wanted acknowledgement or verification of her experience from others; and

5) Kate looked to authority figures to take charge and protect her, thus abdicating her responsibility to others

Pienkos observes that Kate experienced herself as threatened and invaded by hostility, being responsible for protecting others from this hostility, but being unable or unwilling to manage it on her own. Pienkos goes on to posit that the conflict then arises that others are endangered by her hostility, but others are also the only means of protecting her from it. Pienkos notes this suggests conflict around "ownership and responsibility" of aggression, "whom the aggression belongs to, ... and who is capable of handling it." (2014, p. 265). We tend to agree with this viewpoint, which adds to Westerman's caveat that aggression and autonomy are separate drives. Rather than mere expression of aggression, the "ownership of and responsibility of aggression” is more representative of autonomous and mature emotional regulation. 
In regards to psychodynamic therapy, Pienkos notes that the therapist holding an interpretive stance could maintain an authoritarian/power position over Kate, thus fostering dependence rather than independence. Though this is possible, we contend that psychodynamic interpretation ideally serves to provide information to the patient, and does not dictate or suggest decision-making. Just as Westerman posits that expression of anger without purpose is not autonomous, perhaps neither is decision-making without appropriate information. The therapist's goal, in a psychodynamic sense, was to provide an environment in which Kate felt more free and able to practice autonomy and independence than she did elsewhere. Pienkos does note that Kate's reluctance to accept an authority figure's version of reality over her own makes sense in the context of feeling her experience was not validated by her parents. Kate may have experienced validation from therapy that she felt was denied to her previously, although this idea would suggest focusing more on supportive therapy rather than on psychodynamic interpretive therapy. We do acknowledge that there was little discussed in our original case study regarding transference and countertransference issues between Kate and the therapist, and we agree that from a psychodynamic perspective these themes should have been disccussed more in the case study.

\section{WHY HALLUCINATIONS?}

Another important issue that was discussed by several of the commentaries was the potential significance of the hallucinations themselves. One of the concerns raised by Westerman was that excessive focus/significance was placed on the symptom of hallucinations and making a DSM diagnosis, which may have been too limiting in attempting to describe Kate's condition.

However, at least initially, the hallucinations were the focus of treatment because there were no other reported symptoms, and the hallucinations were initially the only reason Kate and her family sought treatment. Further, Kate herself was at some point focused on the diagnosis as to whether or not she had schizophrenia, which as Pienkos noted, may have been part of Kate's conflicted desire to be ill and to be taken care of by authority figures. Kate's parents were also concerned about the possibility of a diagnosis of schizophrenia because it had important implications for prognosis, course, longevity, and treatment.

We understand the downsides of having to focus on what are essentially labels that fail to fully encapsulate a complex dynamic process which is affecting not only an individual, but is clearly rooted in family systems. However, this conversation about diagnosis must still be had when patients and families inquire about length of treatment, medication management, and longevity of symptoms. Additionally, a biological perspective should involve a ruling out of what are considered to be more serious, severe, and protracted illnesses, which include schizophrenia and organic syndromes such as dementia and delirium. As Mertin notes, an inappropriate diagnosis of schizophrenia would continue with the child into the medical record, and would likely influence all future evaluations and clinical decision-making when reviewed by other physicians or mental health professionals. In sum, we felt it imperative to rule out the diagnosis of schizophrenia.

We also believe it is important to make a distinction between focusing on the hallucinations in a conceptual formulation/diagnosis, and focusing on the hallucinations in 
treatment with Kate. As the hallucinations were felt to be achieving for Kate some primary and/or secondary gain as well as masking her true internal conflicts, the therapist did guide Kate's parents to not focus on the hallucinations, and as much as possible, hallucinations were not the focus of individual treatment with Kate. However, the therapist would at times casually ask about the hallucinations as a way to mark how Kate was handling her own internal conflicts, but the hallucintions were not viewed as signifying worsening psychopathology.

Since we considered the diagnosis of conversion disorder, we paid some attention to whether primary gain existed in regards to the hallucinations. As Westerman points out, there is a false dichotomy of "problems that are and are not functional in nature" (2014, p. 273) , as any symptom could really serve both purposes, including true symptoms of psychosis. We paid special attention to this as, by definition, a conversion disorder requires the symptoms serve a functional purpose. If significant sources of primary gain could not be elucidated, perhaps this would have pointed away from suspecting the symptoms to be of a conversion disorder nature.

What do the hallucinations signify? Pienkos notes that auditory hallucinations can be viewed as "meaningful responses to life circumstances" (p. 267) and are core components to understanding a patient's central conflicts rather than merely symptoms of a psychiatric disorder. Pienkos further states that it is difficult to classify hallucinations without obtaining a thorough understanding of their essential features. Pienkos posits that rather than discerning psychotic from non-psychotic hallucinations, there exists a continuum of hallucinations on a spectrum; however, it is still difficult to discern which phenomena occur in true psychosis and which occur outside of psychosis, and whether or not these are mutually exclusive. Thus Mertin advocates for breaking the link between hallucinations and psychosis and realizing that they do not refer to identical constructs. Mertin notes that hallucinations have a high discontinuation rate in children and adolescents regardless of whether they receive mental health services, and that the presence of hallucinations in early childhood is most often transient and benign, although a variety of factors could be associated with the persistence and severity of hallucinations. One major limitation noted by Mertin is that there are no formal guidelines for the diagnosis of children with hallucinations, which is perhaps in further support of Westerman's view that the hallucinations themselves should be less of the focus in treatment.

Mertin observes that traumatic life experiences are often triggers for auditory hallucinations. The case could be made that Kate's childhood, namely a father struggling with alcoholism that left her mother unavailable to provide optimal emotional support to Kate, contained several traumatic experiences, if not one protracted experience. Ironically, or perhaps more puzzling, is that Kate's hallucinations did not appear during the traumatic experience of her childhood, but only after the perceived trauma was resolved. It was never clear why the hallucinations started so abruptly, although this was a significant diagnostic clue, as symptoms of early-onset schizophrenia (EOS) typically have a more insidious onset.

Mertin also notes that other correlates for non-psychotic hallucinations in children and adolescents include parental psychiatric pathology, including alcoholism, and lack of parental support. Again, it is intriguing that the hallucinations first occurred approximately two years after these family issues may have been considered resolved, rather than the hallucinations occurring when the traumatic experiences were occurring. 
Mertin also notes that high levels of emotion and anxiety can trigger hallucinations in those who have experienced trauma, and that these individuals experience high levels of reexperiencing the trauma. Again, the authors were unable to determine if there was a specific trigger or re-experiencing event that preceded the onset of the hallucinations. Mertin goes on to say that in these cases, "many of the voices were reported as being threatening or distressing" (2014, p. 289) and "the content of the hallucinations was often relevant in understanding the underlying psychopathology in the child's life" (2014, p. 290). These characteristics certainly apply to Kate's hallucinations. Therefore, perhaps initially more attention could have been paid to the psychosocial context in which the hallucinations were occurring, rather than the hallucinations themselves. Having a more thorough understanding of the social and emotional environment in which the hallucinations arose may have enhanced the therapist's ability to understand and treat Kate. Mertin suggests that after a psychotic condition was reliably ruled out, that the hallucinations should be regarded merely as evidence of high levels of emotional stress rather than a specific symptom that needs separate treatment. We agree with this point, and although a great proportion of the initial dynamic formulation and differential diagnosis involved the hallucinations, the individual treatment did not have such a focus, but rather emphasized avoiding focus on the hallucinations when at all possible.

In regards to delusional thinking, Mertin notes that this may occur as a result of the increased arousal and anxiety associated with experiencing hallucinations, thereby being secondary to the hallucinations themselves. Not surprisingly, Mertin notes the strong association between traumatic experiences and both the incidence and persistence of hallucinations, as well as the intrusiveness of the hallucinatory experience, which in turn increases the risk for secondary delusional thinking. Kate's secondary delusional thinking is another reason why carefully ruling out the diagnosis of schizophrenia remained important. As Mertin notes, there is a lack of evidence suggesting that secondary delusional thinking is a risk for more serious psychopathology. However, it remains important to determine whether the delusional ideation is in fact a result of the distress associated with experiencing the hallucinations, or whether this represents a genuine thought disorder associated more with a psychotic condition.

The question of why some children and adolescents experience auditory hallucinations while others do not remains largely unexplained. It is possible that children who experience hallucinations are simply more emotionally vulnerable to high levels of stress and have poorer coping skills. As discussed by both the commentators and us, Kate's search for greater autonomy and independence was a major inciting factor in her presentation and symptomology. However, we would all would have to agree that most adolescents who strive for autonomy and independence from their parents do not experience hallucinations. Likewise, most adolescents with parents who struggle with alcohol or drug addiction do not experience hallucinations. And while some adolescent "acting out" may be expected, it remains unclear why an unconscious mechanism that produced quasi-psychotic symptomology was Kate's way of acting out. Thus, the question of why Kate started hallucinating at the particular time that she did, rather than at some other time, or rather than developing some other symptom, remains unanswered. 


\section{SUMMARY}

We reviewed the case study of Kate, a 16-year-old young woman who initially presented with auditory hallucinations that we felt to be non-psychotic. The commentaries by Mertin, Pienkos, and Westerman provided additional insight regarding the nature of these symptoms, the underlying mechanisms that produced these symptoms, and the theoretical underpinnings that may best explain and successfully treat these symptoms. However, more importantly, the commentators and we agree that focusing merely on the symptoms bypasses the importance of this case-namely, that a more thorough understanding of complex unconscious processes which exist between a child and family need to be understood when taking such symptoms into larger context. The commentators and we, while coming from different theoretical backgrounds, all seem to advocate for greater attempts at understanding individual and family dynamics, and not relying solely on symptoms and labels in formulating case conceptualizations and treatment plans. Certainly, this case highlights the need to look at each patient individually and to understand that symptoms in children and adolescents exist in the context of a larger family dynamic, the understanding of which may be integral to truly helping children and their families.

\section{REFERENCES}

Mertin, P. (2014). What do we know about the correlates and underlying causes of auditory hallucinations in nonpsychotic children and adolescents, and what are the implications for diagnosis and treatment? Pragmatic Case Studies in Psychotherapy, 10(4), Article 4, 287-295. Available: http://hdl.rutgers.edu/1782.1/pcsp_journal

Pienkos, E. (2014). Using phenomenology to understand hallucinatory experiences. Pragmatic Case Studies in Psychotherapy, 10(4), Article 2, 260-270. Available: http://hdl.rutgers.edu/1782.1/pcsp_journal

Shapiro, M.A., Bussing, R., \& Nguyen, M.L. (2014). Pseudohallucinations in an adolescent: Considerations for diagnosis and treatment in the case of "Kate." Pragmatic Case Studies in Psychotherapy, 10(4), Article 1, 227-259. Available: http://hdl.rutgers.edu/1782.1/pcsp_journal

Westerman, M.A. (2014). The case of "Kate" and her pseudohallucinations from the perspective of interpersonal defense theory: An example of using case formulations as the key guide for understanding patients' problems and making treatment decisions. Pragmatic Case Studies in Psychotherapy, 10(4), Article 3, 271-286. Available: http://hdl.rutgers.edu/1782.1/pcsp_journal 